\title{
Performance of anti-CD19 chimeric antigen receptor $T$ cells in genetically defined classes of chronic lymphocytic leukemia
}

\author{
Veronika Mancikova (D) , ${ }^{1,2}$ Helena Peschelova, ${ }^{1}$ Veronika Kozlova, ${ }^{1,2}$ \\ Aneta Ledererova, ${ }^{1,2}$ Adriana Ladungova, ${ }^{1}$ Jan Verner, ${ }^{1,2}$ Tomas Loja, \\ Frantisek Folber, ${ }^{2}$ Jiri Mayer, $^{1,2}$ Sarka Pospisilova, ${ }^{1,2}$ Michal Smida ${ }^{1,2}$
}

To cite: Mancikova V, Peschelova H, Kozlova V, et al. Performance of anti-CD19 chimeric antigen receptor T cells in genetically defined classes of chronic lymphocytic leukemia. Journal for ImmunoTherapy of Cancer 2020;8:e000471. doi:10.1136/jitc-2019-000471

- Additional material is published online only. To view, please visit the journal online (http://dx.doi.org/10.1136/jitc2019-000471).

VM, HP and VK contributed equally.

VM and MS are joint senior authors.

Accepted 09 March 2020

\section{Check for updates}

(C) Author(s) (or their employer(s)) 2020. Re-use permitted under CC BY-NC. No commercial re-use. See rights and permissions. Published by BMJ.

${ }^{1}$ Central European Institute of Technology (CEITEC), Masaryk University, Brno, Czech Republic ${ }^{2}$ Department of Internal Medicine-Hematology and Oncology, University Hospital Brno and Faculty of Medicine, Masaryk University, Brno, Czech Republic

\section{Correspondence to}

Dr Veronika Mancikova; veronika.mancikova@ceitec. muni.cz

Dr Michal Smida; michal.smida@ceitec.muni.cz

\section{ABSTRACT}

Background While achieving prolonged remissions in other B cell-derived malignancies, chimeric antigen receptor (CAR) T cells still underperform when injected into patients with chronic lymphocytic leukemia (CLL). We studied the influence of genetics on CLL response to antiCD19 CAR T-cell therapy.

Methods First, we studied 32 primary CLL samples composed of 26 immunoglobulin heavy-chain gene variable (IGHV)-unmutated (9 ATM-mutated, 8 TP53mutated, and 9 without mutations in ATM, TP53, NOTCH1 or SF3B1) and 6 IGHV-mutated samples without mutations in the above-mentioned genes. Then, we mimicked the leukemic microenvironment in the primary cells by ' $2 \mathrm{~S}$ stimulation' through interleukin-2 and nuclear factor kappa B. Finally, CRISPR/Cas9-generated ATM-knockout and TP53-knockout clones (four and seven, respectively) from CLL-derived cell lines MEC1 and HG3 were used. All these samples were exposed to CAR T cells. In vivo survival study in NSG mice using HG3 wild-type (WT), ATMknockout or TP53-knockout cells was also performed. Results Primary unstimulated CLL cells were specifically eliminated after $>24$ hours of coculture with CAR T cells. '2S' stimulated cells showed increased survival when exposed to CAR T cells compared with unstimulated ones, confirming the positive effect of this stimulation on CLL cells' in vitro fitness. After 96 hours of coculture, there was no difference in survival among the genetic classes. Finally, CAR T cells were specifically activated in vitro in the presence of target knockout cell lines as shown by the production of interferon- $\gamma$ when compared with control (CTRL) T cells $(p=0.0020)$, but there was no difference in knockout cells' survival. In vivo, CAR T cells prolonged the survival of mice injected with WT, TP53-knockout and ATM-knockout HG3 tumor cells as compared with CTRL T cells ( $p=0.0485,0.0204$ and $<0.0001$, respectively). When compared with ATM-knockout, TP53-knockout disease was associated with an earlier time of onset $(p<0.0001)$, higher tumor burden $(p=0.0002)$ and inefficient T-cell engraftment $(p=0.0012)$.

Conclusions While in vitro no differences in survival of CLL cells of various genetic backgrounds were observed, CAR T cells showed a different effectiveness at eradicating tumor cells in vivo depending on the driver mutation. Early disease onset, high-tumor burden and inefficient T-cell engraftment, associated with TP53-knockout tumors in our experimental setting, ultimately led to inferior performance of CAR T cells.

\section{BACKGROUND}

Chronic lymphocytic leukemia (CLL) is the most common adult B-cell leukemia characterized by high genetic and clinical diversity. Genetic mutations are major predictor factors of prognosis, time-to-first-treatment, as well as response to treatment in CLL. ${ }^{1}$ For instance, patients with TP53 alterations display poor prognosis with shorter time-to-first-treatment and resistance to chemoimmunotherapy (based on fludarabine, cyclophosphamide and anti-CD20 antibody rituximab) routinely used to manage CLL. ${ }^{2}$ Therefore, these patients are nowadays rather treated with targeted inhibitors (ibrutinib, idelalisib or venetoclax) that show some capacity to induce response in these difficult-to-treat patients. $^{3}$ Apart from TP53 aberrations, several other genetic defects have been associated with aggressive CLL course, including the unmutated immunoglobulin heavy-chain gene variable $(I G H V)$ region and mutations in ATM, SF3B1, and NOTCH1. Ultimately, several factors, including $I G H V$ mutational status, genomic changes, patient age, disease stage and the presence of comorbidities, are used nowadays to select the most appropriate treatment option for each patient. ${ }^{4}$ However, with the exception of allogeneic transplantation, CLL remains incurable.

One possibly curative option could be chimeric antigen receptor (CAR) T-cell immunotherapy. CAR $\mathrm{T}$ cells are prepared by genetic modification of patients' $\mathrm{T}$ cells. Tumor specificity is imposed on these cells by introducing a synthetic gene coding for a receptor composed of an antigen-binding 
domain derived from a B-cell receptor fused to T-cell activation domains (such as CD28 or $4-1 \mathrm{BB}^{5}$ ). This modification reprograms $\mathrm{T}$ cells to target selected antigen on the surface of malignant cells. Since its application in CLL is so far limited to clinical trials, only patients with relapsed and/or refractory $(\mathrm{r} / \mathrm{r})$ disease have been treated with this therapy. Using CAR T cells targeting CD19 has shown durable complete remissions in these heavily pretreated patients, but only in up to $29 \%$ of them. ${ }^{6}{ }^{7}$ In general, such favorable response among patients with CLL is much lower when compared with patients with other $\mathrm{r} / \mathrm{r}$ B-cell malignancies treated with anti-CD19 CAR T cells, where $37 \%-55 \%$ of them reach durable complete remissions. ${ }^{89}$ Some of the possible reasons for this disproportion are inhibitory tumor environment of CLL and larger tumor burdens in patients with CLL at the moment of treatment (reviewed in Lorentzen and Straten ${ }^{10}$ ). Additionally, attributes of the final CAR T-cell product, including T-cell fitness, phenotypical differentiation and metabolic program, impact the ultimate therapeutic outcome. ${ }^{11}$ Apart from these, individual disease-specific characteristics that would distinguish responders from those who will not benefit from CAR T-cell treatment have not been described so far. ${ }^{11}$ However, the CLL clinical trials have been done only with small numbers of patients and could be underpowered to detect some associations. Thus, the impact of individual genetic aberrations on the response of CLL cells to CAR T-cell therapy has not been reliably evaluated.

Herein, we have comprehensively assessed the effect of various clinically relevant mutations on the response of CLL to CAR T cells in several in vitro and in vivo disease models. In vitro, anti-CD19 CAR T cells were similarly effective at eliminating CLL model cell lines and primary CLL cells of various genetic backgrounds. In vivo, CAR T cells were able to prolong survival of all studied genetic backgrounds but with different curative rate, which closely reflected the disease severity and was lowest in the TP53-knockout group.

\section{MATERIALS AND METHODS Human primary samples, cell lines and culture conditions}

Buffy coats from healthy individuals were purchased from the Department of Transfusion and Tissues of University Hospital Brno (UHB). RosetteSep Human T Cell Enrichment Cocktail (StemCell) was used to isolate primary $\mathrm{T}$ cells according to manufacturer's protocol. Thirty-two primary CLL samples were selected from the biobank of the Department of Internal Medicine, Hematology and Oncology, UHB. All patients fulfilled the "International Workshop on Chronic Lymphocytic Leukemia"/National Cancer Institute diagnostic criteria for CLL. ${ }^{12}$ Collected peripheral blood samples were subjected to gradient centrifugation to obtain high-purity B lymphocytes (>98\%) using Ficoll-Paque PLUS (GE Healthcare) combined with RosetteSep Kits (StemCell) to deplete non-B cells and subsequently vitally frozen. The selection criteria were based on availability of samples' genetic information in order to compile genetically defined groups. All selected samples had been screened by Sanger and/or next-generation sequencing for somatic hypermutation in $I G H V^{13}{ }^{14}$ and mutations in $T P 53,{ }^{1516} A T M,{ }^{17}$ NOTCH $1{ }^{18}$ and SF3B $1 .{ }^{19}$ Only cases with highly abundant TP53 and ATM mutations were included. Conversely, wild-type (WT) cases had no mutation detected above the threshold of a respective method used. All primary cells ( $\mathrm{T}$ and CLL cells) were cultivated in serum-free AIM-V medium (Thermo Fisher Scientific). T cells were stimulated by interleukin (IL)-2 (50 U/mL, Miltenyi Biotech) and Dynabeads Human T-activator CD3/CD28 (ratio 1:3, bead:cell; Thermo Fisher Scientific). For '2S' stimulation of CLL cells, resiquimod $(1 \mu \mathrm{g} / \mathrm{mL}$, Sigma $)$ and IL-2 $(500 \mathrm{U} / \mathrm{mL})$ were supplemented to the cells 3 days prior to starting any experiments.

HG3 (a generous gift from Dr Rosenquist,Sweden), MEC1 (DSZM) and Lenti-X 293T (TakaraBio Inc.) cell lines were used herein.HG3 is WT in TP53 gene ${ }^{20}$, while MEC1 has a truncated TP53 allele resulting fromc.949_950insC mutation and the other allele is deleted $^{21}$. Both cell lineshave unmutated IGHV. HG3 was maintained in RPMI-1640 medium (Biosera), MEC1 in Iscove's Modified Dulbecco's Medium (Biosera) and Lenti-X in Dulbecco's Modified Eagle's Medium (Biosera). All media were supplemented with10\% (v/v) heat-inactivated fetal bovine serum (FBS; Biosera) and $1 \%$ (v/v)penicillin/streptomycin (MP Biomedicals).

\section{Lentiviral plasmids, CAR virus production and gene transduction}

Sequence encoding anti-CD19 CAR was previously described $^{22}$ and synthetized in BlueHeron Biotech Company (USA). This construct was subsequently cloned into BamHI and SaII sites of the lentiviral vector pRRL-CMV-GFP-sin 18 (originally generated by D Trono, purchased from Addgene), in which the CMV promotor had been exchanged for EF1 $\alpha$ promotor by molecular cloning. The sequence of each genetic fragment was confirmed by Sanger sequencing. Additionally, helper plasmids pCMV-VSV-G and pCMV-dR8.91 were from Addgene (originally generated by $\mathrm{D}$ Trono).

To generate lentiviral particles, we first treated Lenti-X cells with $25 \mu \mathrm{M}$ Chloroquine Diphosphate (Sigma) 5 hours prior to transfection. Subsequently, we used polyethylenimine (PEI, $1 \mathrm{mg} / \mathrm{mL}$; Polysciences) in a ratio of 3:1 (PEI:DNA) for transfection, where we combined the plasmids in equimolar ratio and used a total of $3 \mu \mathrm{g}$ of DNA per $8 \times 10^{5}$ Lenti-X cells. The medium was replaced 3 hours later. Conditioned lentiviral medium was harvested 48 and 72 hours later, frozen and stored at $-80^{\circ} \mathrm{C}$. Lenti-X Concentrator (TakaraBio) was used to concentrate the conditioned medium according to the manufacturer's protocol. After centrifugation, the pellets were resuspended in AIM-V medium (1/100th of the original volume) and used immediately to transduce target T cells. A $1.5 \mathrm{~mL}$ Eppendorf tube was coated with 
$0.2 \mathrm{~mL}$ of Retronectin (TakaraBio) diluted to $100 \mu \mathrm{g} / \mathrm{mL}$ for 2 hours at room temperature and then incubated with $2 \%$ bovine serum albumin (Sigma) for $30 \mathrm{~min}$. T cells $\left(8 \times 10^{5}\right)$, which were prestimulated with IL-2 and CD3/ CD28 24 hours prior to transduction, were resuspended in the retronectin-coated tube in $0.5 \mathrm{~mL}$ of concentrated virus supplemented with Polybrene $(10 \mu \mathrm{g} / \mathrm{mL}$, Merck) and centrifuged at $1000 \mathrm{~g}$ at $32^{\circ} \mathrm{C}$ for 2 hours. Immediately afterwards, viral medium was replaced. T cells were then left to expand until further use (the total in vitro cultivation time never exceeded 10 days).

\section{Single-cell knockout clones' production and their verification}

MEC1 bears an inherent TP53 impairment. To be able to use it to generate various genetically distinct singlecell clones with either TP53 or ATM knocked out, we first had to reintroduce the TP53 WT allele under an inducible promotor. pCMV-Neo-Bam p53 WT plasmid (\#16434, Addgene) was digested by BamHI, and the digestion product (TP53 WT gene) was cloned into a pLenti CMV/TO Hygro empty plasmid (\#17484, Addgene) under a doxycyclin-inducible promotor. Lentiviral particles were produced as described previously; MEC1 cells were transduced and selected by $500 \mu \mathrm{g} / \mathrm{mL}$ hygromycin (InvivoGen) for 14 days. Afterwards, the lentiGuidePuro two-vector system, composed of single guide RNA (sgRNA) containing lentiGuide-Puro vector (\#52963, Addgene) and Cas9 expressing LentiCas9-Blast vector (\#52962, Addgene), was used to generate CRISPR knockouts of ATM and TP53 genes. The following sgRNAs were cloned into lentiGuide-Puro:

5'-GGATGCTGTTCTCAGACTGA-3' targeting exon 6 of ATM

5'-TCGACGCTAGGATCTGACTG-3' targeting exon 2 of TP53

5'-TATCTGAGCAGCGCTCATGG-3' targeting exon 5 of TP53

Transfection and virus harvesting were performed as described previously. Cas9-transduced cells (HG3 and MEC1 p53 wt) were selected by $10 \mu \mathrm{g} / \mathrm{mL}$ blasticidin (InvivoGen) for 7 days. Immediately afterwards, cells were transduced by lentiviruses containing the sgRNA of interest and selected by $10 \mu \mathrm{g} / \mathrm{mL}$ puromycin (InvivoGen, \#ant-pr-1) for 4 days. In order to obtain monoclonal cell populations, the selected cells were plated into 96-well plates as 1 cell per well using the limiting dilution method.

Sanger sequencing and CRISP-ID tool (http://crispid. gbiomed.kuleuven.be/) were used to detect editing events. In the cell clones harboring indels, the loss of the respective protein was verified by western blotting. Only clones with no band visible after prolonged exposure were selected for further study. For ATM protein detection, no prior stimulation was needed. In case of p53, a 24-hour treatment with $2 \mu \mathrm{M}$ doxorubicin was performed to stabilize the p53 protein. Prior to the treatment with doxorubicin, MEC1 p53 wt cells were also incubated with $2 \mu \mathrm{g} / \mathrm{mL}$ doxycycline for 24 hours in order to induce the
WT p53 expression. In order to assess the accumulation of p21, the knockout cells were incubated with $2 \mu \mathrm{M}$ doxorubicin for 24 hours, lysed and subjected to western blotting. Additionally, the phosphorylation of KAP1 was determined by western blotting in ATM-knockout cells incubated with $1 \mu \mathrm{M}$ doxorubicin for 4 hours. The antibodies used in the study are listed in online supplementary table 1 .

\section{Cytotoxicity in vitro assay and cytokine production assay}

The antileukemic in vitro activity of CAR $\mathrm{T}$ cells was assessed in 4-day cultures using 1:5 ratio of target:effector cells. For this assay, the collection of knockout clones, as well as the set of primary CLL cells, were used as targets. In the case of vitally frozen primary CLL cells, these were thawed, and their viability was assessed by flow cytometry. A part of CLL cells was subsequently $2 \mathrm{~S}$ stimulated and used in cytotoxicity assay only 72 hours later, while the other part was directly mixed with $\mathrm{T}$ cells. In detail, $2 \times 10^{4}$ live $\mathrm{B}$ cells were combined with $1 \times 10^{5} \mathrm{~T}$ cells (control (CTRL) or CAR) in 96-well U-bottom plates in $200 \mu \mathrm{L}$ of AIM-V medium without any supplements. The target B-cell survival was monitored at 24 and 96 hours by flow cytometry. The amount of retrieved CD19+ cells from the mixture of B cells with CTRL T cells was set as $100 \%$ and compared with the amount of CD19+ cells cultured with CAR T cells. Additionally, supernatants from 4-day coculture of knockout cell clones and CTRL/CAR T cells were collected, and production of interferon (IFN)- $\gamma$ was measured by ELISA using Human IFN- $\gamma$ Standard TMB ELISA Development Kit (PeproTech) following manufacturer's protocol.

\section{In vivo studies}

Mouse experiments were carried out with equal numbers of male and female NOD-scid IL2Rg ${ }^{\text {null }}$ (NSG) mice (Charles River Laboratories, France) 8-12 weeks old per experimental group.

To assess the penetrance of individual knockout clones, $2.5 \times 10^{6}$ tumor cells from each individual clone or WT HG3 and MEC1 cells were injected via tail vein in a total volume of $0.1 \mathrm{~mL}$ phosphate-buffered saline. For further studies, we preferentially selected clones derived from the HG3 cell line, which had the most similar disease-onset time. Two experimental settings to assess CAR T-cell in vivo performance were used: low-tumor and high-tumor burden settings. These scenarios differed in the number of tumor cells $\left(5 \times 10^{5}\right.$ cells and $2 \times 10^{6}$ cells, respectively). Moreover, CTRL or CAR T cells were injected intravenously 5 or 7 days later (low or high scenarios, respectively). Mice were closely monitored for any manifestation of the disease and were sacrificed when symptoms of severe disease (lethargy, excessive weight loss, change in fur quality, apathy and hind leg paralysis) occurred. Spleen was harvested, mechanically homogenized and passed through a $30 \mu \mathrm{m}$ nylon filter, and erythrocytes were lysed using Tris-buffered ammonium chloride. Samples were then analyzed by staining with fluorescently conjugated monoclonal antibodies. Blood 
samples from the surviving CAR-treated animals were obtained from the tail vein approximately a week after the mice regained their starting weight. DNA from blood and spleen tissues was extracted using NucleoSpin Tissue DNA extraction kit (Macherey-Nagel) following the manufacturer's instructions.

\section{Detection of integrated CAR transgene by quantitative PCR}

Quantitative real-time PCR was performed on $150 \mathrm{ng}$ of DNA with three replicates for each sample using the $2 \mathrm{X}$ Taqman Gene Expression Master Mix (Applied Biosystems). Specific primers and probe were designed to amplify the junction region between the light and heavy chain of the single-chain variable fragment of anti-CD19 antibody (clone FMC63) that is contained within the CAR construct: Forward primer: 5'-ACAGGGTAATACGCTTCCGT-3' Reverse primer: 5'-CCCTGAGACAGTGCATGTGA-3' MGB probe: 5'-FAM CCACCTGTGATCTCCA GCTTGGTC-3'

PCR with real-time fluorescence detection was performed on a 384-well Thermo Scientific QuantStudio 12k (Thermo Fisher Scientific). The number of copies for each vector, expressed as copies/ng DNA, was determined by comparison of the measured cycle threshold for each well to the cycle threshold of a standard curve prepared by dilution of a linearized receptor-encoding plasmid spiked into $150 \mathrm{ng}$ of CTRL mouse genomic DNA (obtained from a healthy NSG animal) per well. The $\mathrm{R}^{2}$ value of the standard curve was 0.9945 , and the slope was -3.4134 (corresponding to efficiency of reaction of $96 \%$ ). Known spike controls ranged from $1 \times 10^{1}$ to $1 \times 10^{7}$ copies per well.

\section{Flow-cytometric analysis}

All flow-cytometric analyses were performed using FACS Verse flow cytometer (BD Biosciences). Antibodies used in the study are listed in online supplementary table 1 . To confirm the CAR expression, double staining was used: biotinylated goat antimouse polyclonal IgG antibody (Thermo Fisher Scientific) was visualized with AlexaFluor647-conjugated streptavidin (Invitrogen). In all flow-cytometric analyses, cell viability was monitored with 7-aminoactinomycin D (Invitrogen). Data were analyzed in FlowJo V.10 software.

\section{Statistical analysis}

All statistical analyses were performed using software incorporated in GraphPad Prism V.5. Specific statistical tests used for different study variables are described in the figure legends. All tests were two-sided. $\mathrm{P}$ values of $<0.05$ were considered statistically significant.

\section{RESULTS}

\section{In vitro anti-CD19 CAR T cells are similarly effective at} eliminating primary CLL cells of various genetic backgrounds

For the present study, we selected the anti-CD19 CAR incorporating 4-1BB costimulation molecule in the design. ${ }^{52}$ Our optimized protocol led to the production of a high yield of CAR-expressing T cells $(>85 \%$, online supplementary figure 1A). The functionality of CAR T cells was validated in various in vitro tests (online supplementary figure 1B). In order to comprehensively assess the prognostic value of CLL genetic background on the response to CAR T-cell therapy, we compiled a set of genetically defined 32 primary CLL samples. Of these, 26 were IGHV unmutated (9 ATM-mutated, 8 TP53-mutated, and 9 without mutations in ATM, TP53, NOTCH1 or SF3B1) and 6 bore hypermutation in the IGHV gene but no mutations in the other studied genes. After thawing, no difference in cell viability was observed among the genetic classes (online supplementary figure 2). B cells were then either directly mixed with $\mathrm{T}$ cells or stimulated through IL-2 and nuclear factor kappa B-activating signals ${ }^{23}$ prior to exposure to $\mathrm{T}$ cells in order to mimic a leukemic microenvironment and thus increase the in vitro fitness. After 96 hours of cocultivation, while there still were viable unstimulated CLL cells surviving in culture with CTRL $\mathrm{T}$ cells, no viable unstimulated $\mathrm{B}$ cells were detected in the mixture with CAR T cells (figure 1A). 2S stimulation proved to have a positive effect on CLL in vitro fitness, as some CLL cells survived even in coculture with CAR $\mathrm{T}$ cells (figure 1B). However, after 96 hours of coculture, no significant differences in the survival of the different genetic classes were observed (mean percentage of surviving unmutated IGHVWT cells, 2\%; TP53-mutated, 1.5\%; ATM-mutated, 3.3\%; and IGHV-mutated WT cells, $3.2 \%$; figure 1B). Unsurprisingly, exposure to CAR T cells led to a gradual decrease in the number of target CLL cells throughout the cocultivation period in both studied set-ups (figure 1C).

\section{CRISPR/Cas9 generated TP53-knockout and ATM-knockout single-cell clones recapitulate the results from primary CLL cells in vitro}

Primary CLL cells strive in vitro and, so far, no successful strategy for their reliable xenotransplantation has been described. Therefore, we used the CRISPR/Cas9 system to generate a collection of cell lines with a specific genetic background from commercially available CLLderived cell lines (HG3 and MEC1). We aimed to introduce TP53 or ATM mutations that would result in disruption of the targeted gene expression and thus to generate a set of genetically distinct knockout singlecell clones. However, MEC1 cells already express a truncated p53. Since constitutive WT p53 introduction into p53-defficient tumors could induce senescence ${ }^{24} 25$, we introduced WT TP53 under an inducible promotor. Short-term expression of WT p53 protein could be induced by treating the cells with doxycycline, and it was further stabilized upon DNA damage triggered by doxorubicin (online supplementary figure 3A). These cells were denoted as 'MEC1 p53 WT' and used subsequently to generate individual MEC1 ATM-knockout and TP53-knockout clones. In total, we were able to create 7 TP53 single-cell knockout clones (three and four, respectively) and four ATM single-cell knockout clones (three and one, respectively) from both HG3 and MEC1 cells. As shown in figure 2A, no expression 
A

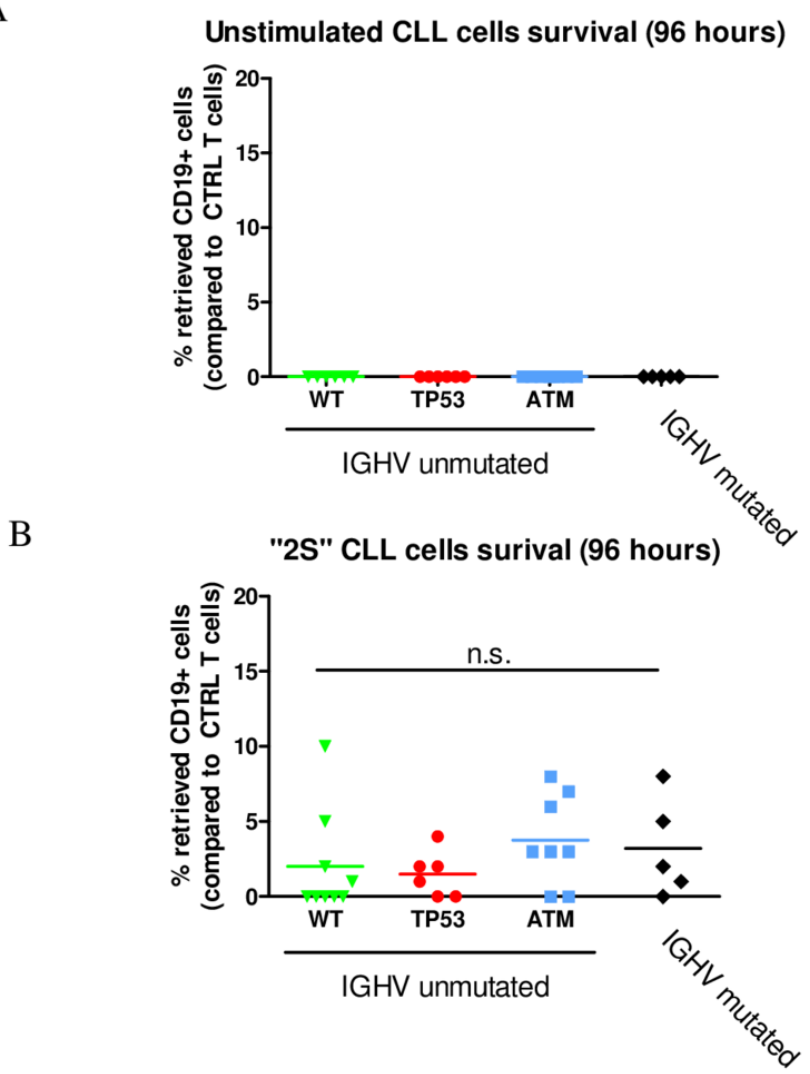

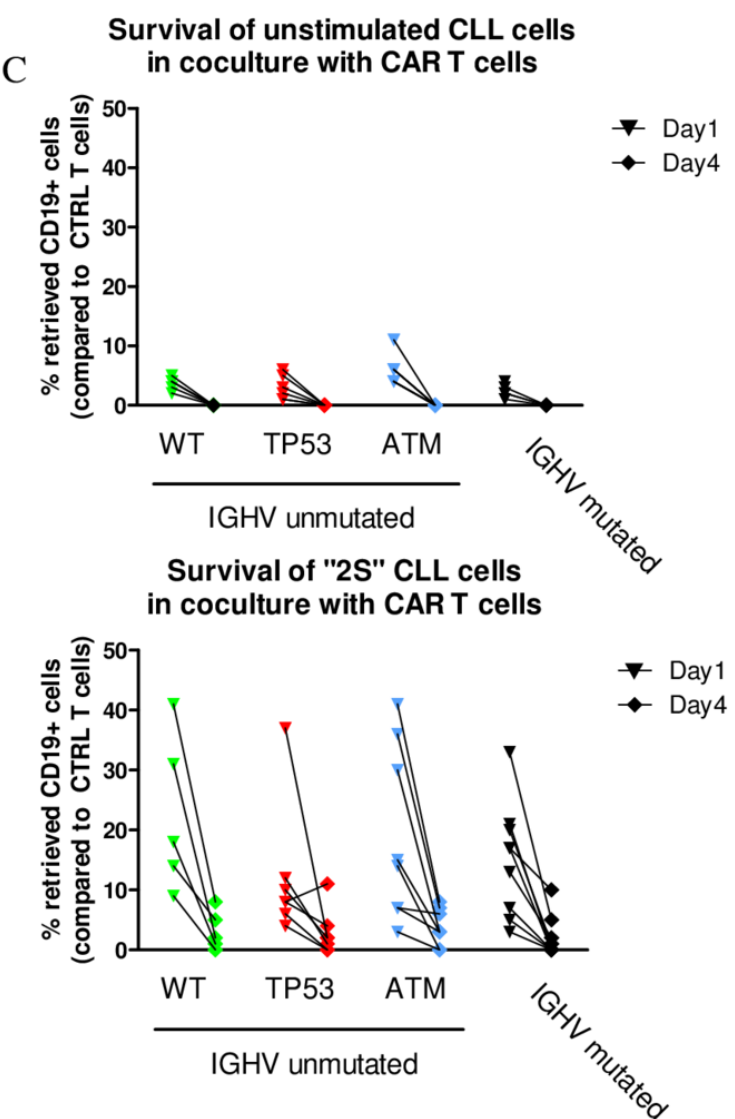

Survival of unstimulated CLL cells

Figure 1 Cytotoxicity of anti-CD19 CAR T cells against primary CLL cells. (A) T cells from a single donor were transduced and mixed with primary unstimulated CLL cells of various genetic backgrounds in 1:5 target:effector ratio. Target cells are color-coded as follows: IGHV-unmutated samples that had an ATM mutation are in blue; those with a TP53 mutation are in red; and those without mutations in ATM, TP53, NOTCH1 or SF3B1 are in green. IGHV-mutated samples without mutations in the above-mentioned genes are in black. This color coding is maintained throughout the manuscript. Cytotoxicity was measured by flow cytometry at 24 and 96 hours of coculture. Results are expressed as percentage of CD19+ leukemia cell recovery after 4 days of culture relative to culture with CTRL T lymphocytes. (B) Primary CLL cells of various genetic backgrounds stimulated with interleukin-2 and nuclear factor kappa B were mixed with CAR/CTRL T cells in 1:5 target:effector ratio and cytotoxicity is measured as in A. (C) Target elimination was gradual over the cultivation period. CAR, chimeric antigen receptor; CLL, chronic lymphocytic leukemia; CTRL, control; IGHV, immunoglobulin heavy-chain gene variable; n.s., not significant; WT, wild type.

of p53 was detectable upon induction of DNA damage in the TP53-knockout cells, and there was a complete loss of ATM protein in the generated knockouts. Even the downstream effector genes were not activated in the generated cell lines upon adequate stimulus (p21 in case of TP53 knockouts, p21 and pKAP1 in ATM knockouts; online supplementary figure $3 \mathrm{~B}$ ), demonstrating true functional impairment of the targeted genes and their downstream signaling pathways in our generated cell lines. In vitro, no other obvious phenotypical changes were associated with acquiring the desired gene mutations; no major differences in cell proliferation were noted (online supplementary figure $3 \mathrm{C}$ ); and no differences in expression of serine protease inhibitor PI-9, an inhibitor of granzyme B-mediated T-cell cytotoxicity, were found (online supplementary figure 3D).

When mixed with available knockout cell lines, CAR T cells, but not CTRL T cells, became specifically activated in their presence as evidenced by the production of IFN- $\gamma$ $(p=0.0020$, figure 2B). In line with previous observations from primary CLL cells, after 96 hours of in vitro coculture with CAR T cells, a similar proportion of CD19+ target cells were surviving in the two genetically defined groups (mean percentage of surviving tumor cells was $12.9 \%$ and $14 \%$ in TP53 and ATM knockouts, respectively; figure 2C). However, when we examined the target elimination in time, we observed that TP53-knockout clones more often exhibited the ability to maintain proliferation in coculture with CAR T cells than their ATMknockout counterparts $(4 / 7$ TP53 clones vs $1 / 4$ ATM clone, figure 2D).

\section{CAR T cells prolong survival of all studied genetic classes in vivo, but with different curative rates}

Since primary CLL cells engraft inefficiently in immunocompromised mice, we chose to evaluate the in vivo efficacy of anti-CD19 CAR T-cell therapy in NSG mice injected with CRISPR/Cas9 generated knockout cell lines. First, we evaluated the kinetics of the disease of all generated cell lines as well as the original HG3 
A

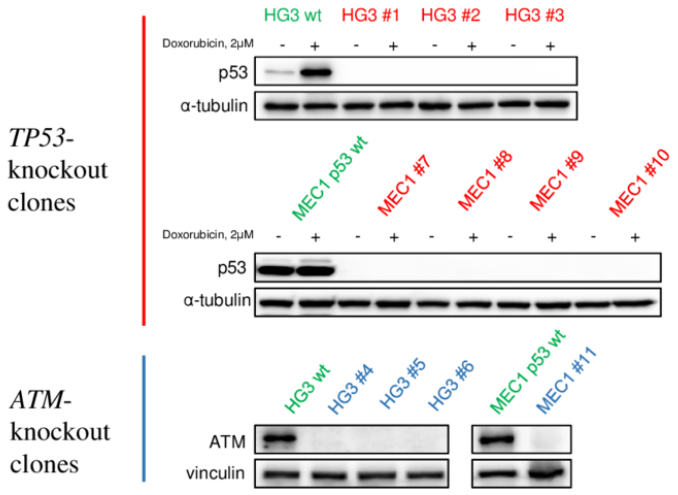

B

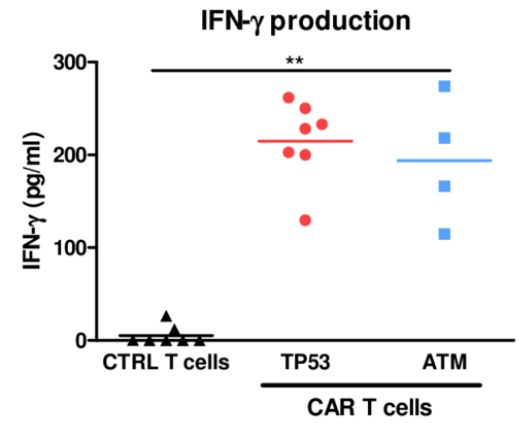

CAR T cells
$\mathrm{C}$

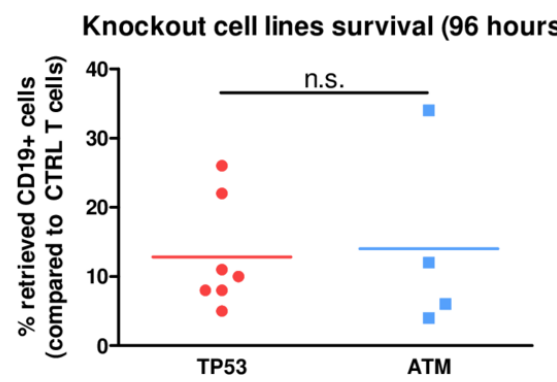

$\mathrm{D}$

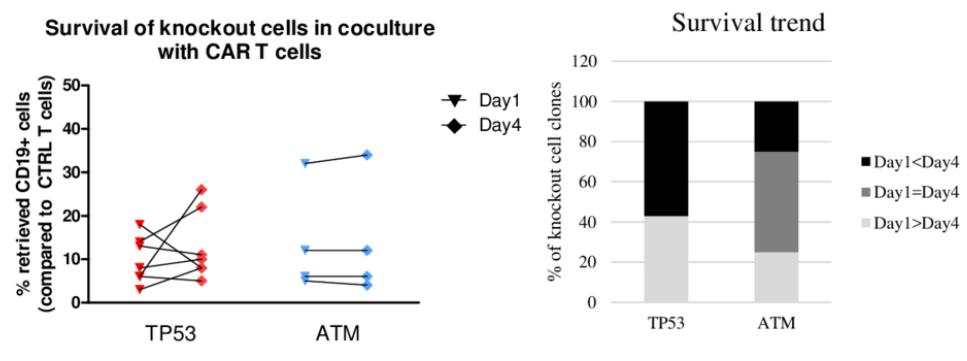

Figure 2 Cytotoxicity of anti-CD19 CAR T cells against cell lines with specific genetic background. (A) Indel mutations were induced in TP53 (in red) and ATM (in blue) genes of HG3 and MEC1 cell lines using CRISPR/Cas9. These mutations resulted in loss of the respective protein as demonstrated by western blotting. (B) Production of IFN- $\gamma$, as measured by ELISA, was significantly induced when CAR T cells were cultured with ATM-knockout and TP53-knockout cell lines as compared with CTRL T cells (Kruskal-Wallis test, $\mathrm{p}=0.0020$ ). (C) Cytotoxicity of CAR T cells against generated TP53-knockout and ATM-knockout cell lines was measured in a 96 hours of coculture by flow cytometry. Results are expressed as percentage of CD19+ leukemia cell recovery after 4 days of culture relative to culture with CTRL T lymphocytes. (D) Target elimination over the cultivation period differed between TP53-knockout and ATM-knockout clones. Fifty-seven percent of TP53-knockout cells were able to proliferate in coculture with CAR T cells contrasted with $25 \%$ of ATM-knockout cells. CAR, chimeric antigen receptor; CTRL, control; IFN, interferon; n.s., not significant.

and MEC1 cells in vivo (figure 3A, HG3 cell line and knockout clones derived from it are depicted with off-color shapes). In this model system, intravenous injection of tumor cell lines led to development of progressive leukemia with significant involvement of the spleen, liver and kidneys. Two HG3-derived TP53knockout clones and an additional ATM clone did not engraft (clones 2, 3 and 6, respectively) even though these mice were followed for 60 days. In the remaining cases, the kinetics of TP53-knockout-driven disease clearly showed a more severe course than the ATMknockout one (median days of succumbing to the disease were 19 and 27, respectively; $\mathrm{p}=0.0262$ ), similar to the original cell lines (median day 20).

We selected HG3-derived clones 1 (TP53 knockout) and 4 (ATM knockout) for further in vivo studies. First, we inoculated NSG mice with $2 \times 10^{6}$ tumor cells (this setting was termed 'high-tumor burden'; figure 3B). Seven days later, CAR or CTRL T cells were injected via tail vein. On day 14, mice injected with the TP53-knockout clone started showing signs of the disease, which was quickly progressing in the following days (figure 3C). In line with the kinetics experiment, ATM-knockout disease onset was delayed (figure $3 \mathrm{C}, \mathrm{p}<0.0001$ ), and its progression was slower. At the moment of succumbing to the disease (median days 16 and 23 in TP53 and ATM CTRL mice, respectively), there was no significant difference in the disease morbidity among the animals injected with CTRL $\mathrm{T}$ cells from the two genetic classes (assessed here by weight loss, figure 3D). However, mice injected with the TP53-knockout clone had a significantly higher tumor burden compared with the ATM knockout $(\mathrm{p}=0.0002$, figure 3D). Due to these disease-related differences, the positive treatment effect was solely apparent in mice with ATM-knockout disease $(\mathrm{p}<0.0001$, figure 3E). Quantification of the integrated CAR-expressing vector in spleens of the treated mice additionally uncovered inefficient engraftment of CAR T cells in the animals with TP53knockout tumors, plausibly caused by shorter time for their proliferation in these mice $(\mathrm{p}=0.0012$, figure $3 \mathrm{~F})$. Eighty-five percent of mice bearing ATM-knockout disease treated with CAR $\mathrm{T}$ cells regained their original weight, were considered cured and afterwards had high levels of CAR T cells detectable in their circulation (figure 3F).

Finally, in an attempt to alleviate the disease burden caused by the TP53 knockout, we inoculated NSG mice with $5 \times 10^{5}$ tumor cells (setting termed 'low-tumor burden') and started the treatment 5 days later (figure $4 \mathrm{~A}$ ). In this 
A

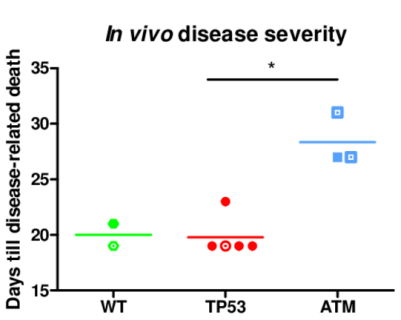

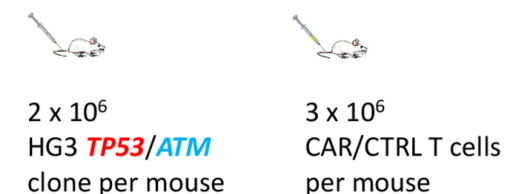

clone per mouse

$\mathrm{C}$

Morbidity

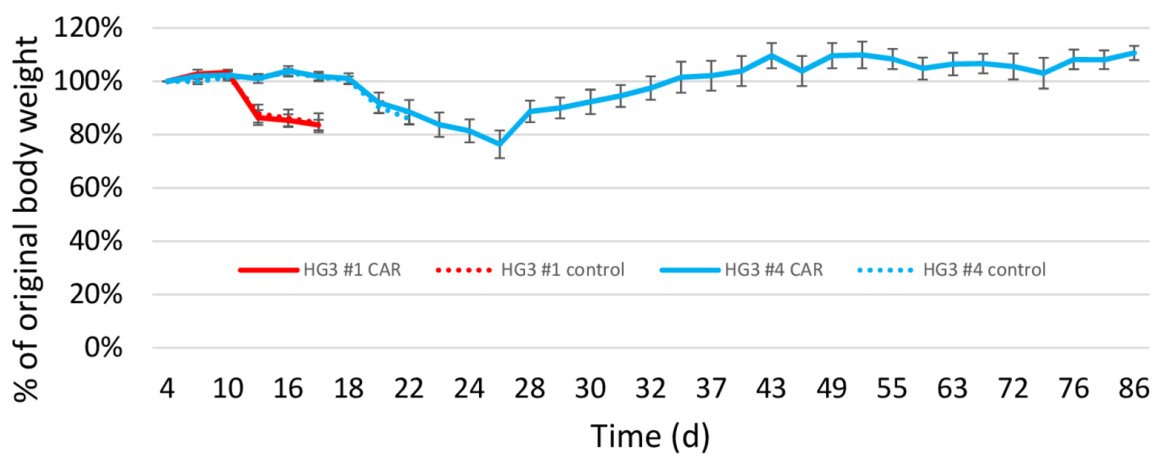

D
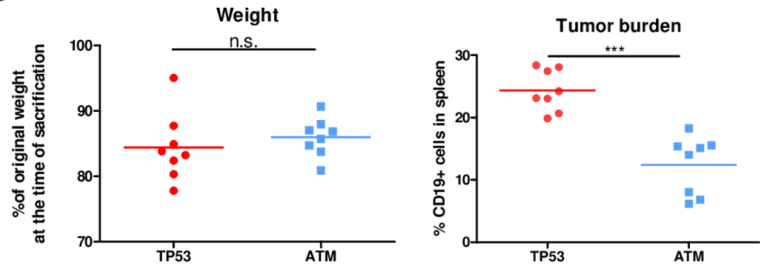

$\mathrm{E}$
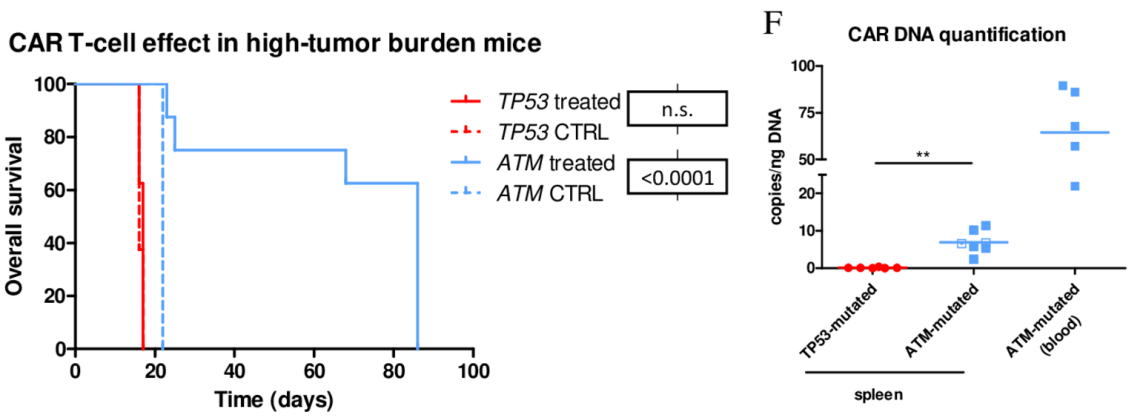

Figure 3 TP53-knockout and ATM-knockout clones induce differently severe progressive leukemia in immunodeficient NSG mice. (A) To assess the penetrance of individual knockout clones, $2.5 \times 10^{6}$ knockout tumor cells or WT HG3 and MEC1 cells were injected intravenously into NSG mice. HG3 and cell lines derived from it are depicted in off-color shapes. Time of diseaserelated death differed significantly between TP53-knockout and ATM-knockout cell lines (Mann-Whitney test, $\mathrm{p}=0.0262)$. (B) Experimental set-up of high-tumor burden setting showing timing and amount of intravenously transferred tumor and CAR/ CTRL T cells in NSG mice. (C) Morbidity was assessed by regular weighting of the animals. It is possible to appreciate from this graphics that TP53-knockout disease develops earlier (Mann-Whitney test, $\mathrm{p}<0.0001$ ) and shows a more aggressive course than ATM-knockout one. (D) No significant difference in the disease morbidity was observed among mice injected with CTRL T cells belonging to either TP53 or ATM group at the time of sacrification (left panel; day of sacrification 17-18 or 23, respectively; Mann-Whitney test; $\mathrm{p}=0.2786$ ). Animals with TP53-knockout disease showed significantly higher tumor burden, as assessed from the number of CD19+ cells detected in spleens of the deceased animals (Mann-Whitney test, $p=0.0002)$. (E) Kaplan-Meier curves showing survival of mice. $P$ values were calculated with two-tailed log-rank Mantel-Cox tests. $(F)$ The number of copies of anti-CD19 CAR vector in spleen and blood DNA from CAR treated mice. Spleens from TP53-knockout mice were harvested at their sacrification due to progressive disease (days 17 and 18), as well as spleens from two ATM-knockout animals (days 24 and 26, in the graphics depicted in off-color). Spleens from remaining mice with ATM-knockout disease were obtained at the termination more than 6 weeks later. Blood samples were obtained from cured animals approximately 1 week after regaining their original weight (day 29). Significantly more copies of CAR vector were detected in spleens of mice with ATM-knockout disease (Mann-Whitney test, $\mathrm{p}=0.0012$ ). CAR, chimeric antigen receptor; CTRL, control; n.s., not significant; NSG, NOD-scid IL2Rg ${ }^{\text {null }}$; WT, wild type. 
A

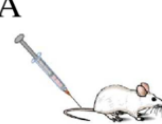

$5 \times 10^{5}$

HG3 WT/TP53/ATM cells per mouse

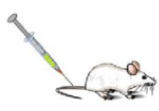

$2 \times 10^{6}$

CAR/CTRL T cells per mouse

B

Figure 4 CAR T-cell treatment prolongs the survival of all genetic groups, but with different curative rates. (A) Experimental set-up of low-tumor burden setting showing the timing and amount of intravenously transferred tumor and CAR/CTRL T cells in NSG mice. (B) Morbidity was assessed in the study by regular weighting of the animals. Even in this setting, disease resulting from injecting HG3 WT and TP53-knockout cells develops early and shows a more aggressive course when compared with ATM-knockout disease. (C) Kaplan-Meier curves showing survival of mice. $\mathrm{P}$ values were calculated with two-tailed log-rank Mantel-Cox tests. CAR, chimeric antigen receptor; CTRL, control; WT, wild type.

setting, mice injected with TP53-knockout cells started showing signs of the disease only 1 day later than in the above-mentioned high-tumor burden scenario (day 15, figure 4B). Similarly, severe disease course was observed among mice injected with HG3 WT cells. On the other hand, the low-tumor cell dose was suboptimal to induce leukemia with $A T M$-knockout cells, since only $4 / 7$ of animals injected with CTRL T cells developed the disease. Nevertheless, in this setting, the treatment partially prolonged survival of animals with TP53-knockout disease ( $\mathrm{p}=0.0204), A T M$-knockout disease $(\mathrm{p}=0.0217)$, as well as those injected with HG3 WT tumor cells $(\mathrm{p}=0.0485$, figure 4C). One of five (20\%) mice injected with HG3 WT cells and treated with CAR T cells was considered cured of disease due to regaining of the original weight and high levels of CAR T cells in blood (assessed by flow cytometry, online supplementary figure 4 ).

\section{DISCUSSION}

In the current era of precision medicine, medical decisions are being tailored to the individual patient based on their predicted response. One example of such personalization from hemato-oncological practice is the preferential treatment of patients with CLL who test positive for aberrations affecting the TP53 gene, with targeted inhibitors $^{3}$ due to their predicted chemorefractoriness. ${ }^{26}$ However, no such personalization is possible so far in the case of CAR T-cell therapy, even though roughly only one out of three patients with CLL will respond to this treatment. ${ }^{67}$ Herein, we set out to comprehensively assess for the first time the impact of two major genetic aberrations on the performance of CAR T cells in several CLL disease models. Not only do we study CAR T-cell performance on a genetically defined set of primary samples, but we also use CRISPR/Cas9 system to prepare several TP53-gene and ATM-gene knockout clones from two CLLderived cell lines. While we do not observe any significant differences in survival of target CLL cells in vitro, in our in vivo disease model, TP53 knockout is associated with earlier time of disease onset, higher tumor burden and inefficient T-cell engraftment, which jointly negatively influence the treatment outcome.

CLL is a disease of many genetic drivers, some of which exhibit oncogenic properties, while others function as tumor suppressors. ${ }^{27}$ The study of disease characteristics and response to treatment using primary CLL cells is hampered by their decreased in vitro viability and their inefficiency to engraft in immunodeficient mice (reviewed in Bertilaccio et $a l^{28}$ ). Nevertheless, in the current study, we worked with a set of genetically characterized primary CLL cells to assess their response to CAR T-cell treatment. All unstimulated CLL cells were efficiently and completely eradicated by CAR $\mathrm{T}$ cells at the end point of our experiment, while some of those in which we mimicked microenvironment interactions ${ }^{23}$ survived the cultivation period. However, the total CLL survival was low $(<5 \%)$. Therefore, to exclude that the results were affected by the reduced fitness of CLL cells in 
prolonged in vitro cultures, we decided to prepare genetically homogeneous CLL-derived cell lines bearing clinically relevant mutations. Herein, we focused on TP53 and ATM genes for several reasons. First, they are among the most frequently mutated genes in CLL. ${ }^{29}$ Second, aberrations in these two genes have been linked with poor prognosis. ${ }^{230}$ Finally, these genes are tumor suppressors (ie, loss or reduction in their function is found in cancer), and thus we could use CRISPR/Cas9 technology to disrupt the gene rather than to introduce any specific mutations, which is technically less challenging. ${ }^{31}$ Of note, while the survival of the generated knockout cell lines in response to CAR T cells was higher (mean $15 \%$ ), we still could not detect any difference in survival according to the genetic background, corroborating the results obtained with primary CLL cells.

The commercial HG3 cell line, which is one of those we used to prepare knockout cells, has been described to be composed of subclones and thus to be heterogeneous. ${ }^{32}$ Therefore, creation of isogenic cell lines originating from a single cell could lead to opportunistic selection of a phenotype associated with one of the subclones rather than with the generated genetic feature. Indeed, when we transplanted our knockout cell line collection into NSG mice, three clones derived from HG3 cells did not engraft, which could possibly point to them being derived from a specific common subclone. Nevertheless, the remaining clones (both TP53 and ATM knockouts) derived from HG3 cells behaved remarkably similarly to those derived from MEC1 cells in all remaining assays. Therefore, we believe that in these clones, the phenotypes we observe are indeed caused by the introduced mutations and not by a subclonal origin of the cells.

Already the in vitro data hinted the aggressive potential of TP53-knockout cell lines, since they more often maintained the ability to proliferate in coculture with CAR T cells. Still, in vitro modeling does not allow for faithful recapitulation of the disease pathogenesis and the complex in vivo relationships between leukemic cells and the microenvironment, ${ }^{28}$ which are crucial in CLL. In our in vivo model, we could recapitulate some of the clinical characteristics of TP53-mutated CLL. For instance, NSG mice injected with TP53-knockout clones showed the shortest time to disease manifestation, which is in accordance with TP53-mutated patients displaying shorter time to first treatment. ${ }^{26}$ Of note, TP53-knockout clones' superior proliferation rate was only apparent in vivo, once again underpinning the positive effect of microenvironment on CLL tumorigenesis. In our model, this aberration was therefore also associated with a higher tumor burden, which has already been connected with failure of the CAR T-cell therapy. ${ }^{33}$

It is important to acknowledge here that some patients with CLL with TP53-mutated disease have already been successfully treated with CAR T-cell therapy. ${ }^{34}$ While in our in vivo experimental setting we worked with a welldefined homogeneous model, in the clinical practice, there is a certain degree of heterogeneity among patients with CLL with TP53-mutated disease: some patients with this poor prognosis marker do not suffer from such a quickly progressing disease and can therefore be candidates for CAR T-cell therapy. Similarly, there are patients who do not harbor TP53 aberrations and suffer from an aggressive progressive disease. ${ }^{35}$ We hypothesize that those could also benefit to a lesser extent from CAR T cells.

Unexpectedly, disease onset in mice injected with ATMknockout cell lines showed major retardation. ATM plays an important role in double-stranded DNA repair, as well as p53 activation with subsequent cell cycle arrest and/or apoptosis. Thus, its inactivation can cause p53 dysfunction. The ATM gene is localized on the long arm of chromosome 11 in a region, which is very frequently deleted in CLL. Those patients with CLL, whose residual ATM allele is mutated, show poorer clinical outcome. ${ }^{36}$ In all of our knockout clones, both alleles of ATM gene were affected by indel mutations, which resulted in complete loss of ATM at protein level. Thus, adverse phenotype was anticipated. However, a similarly mild phenotype as we describe here for ATM knockout has been observed in glioma tumorigenesis, where knockout or inhibition of ATM function promoted animal survival as compared with WT cells. ${ }^{37}$ Similarly, in vivo tumor formation was impaired by $A T M$ knockout in a breast cancer cell line as compared with the WT CTRL. ${ }^{38}$

Finally, the quality of patient's T cells used to prepare the final CAR T-cell product can impact the treatment efficacy in CLL. ${ }^{11}$ In our in vivo models, we did observe inefficient engraftment of CAR $\mathrm{T}$ cells in mice with TP53-knockout-driven disease. However, the CAR T cells for each of the different stages of the study (in vitro studies, high-tumor and low-tumor in vivo burden) were produced from a single healthy T-cell donor (different for every stage) with a similarly high efficiency. Thus, it is highly improbable that any inherent T-cell defects could be affecting our results. The effect we describe is possibly caused by the shorter time $\mathrm{T}$ cells had for proliferation in mice with TP53-knockout-driven tumors. CAR T-cell cytotoxicity has been shown to be blocked by production of high levels of the serine protease PI-9/serpin-6 that irreversibly inactivates granzyme $\mathrm{B} .{ }^{39}$ However, we did not observe any differences in expression of this enzyme among the used cell lines, which could explain the differences we observe in vivo. Further studies could uncover if additional mechanisms (eg, immunosuppressive microenvironment) are also at play in these mice.

\section{CONCLUSIONS}

This is the first study comprehensively assessing the impact of various clinically relevant genetic aberrations on performance of CAR $T$ cells in CLL. In vitro, antiCD19 CAR T cells are similarly effective at eliminating CLL model cell lines and primary CLL cells of various genetic backgrounds. In vivo, early disease onset, hightumor burden and inefficient T-cell engraftment, which 
were associated with TP53-knockout-driven tumors in our experimental setting, ultimately lead to poorer response to CAR T-cell treatment in this CLL subgroup.

Acknowledgements The authors thank Karla Plevova, $\mathrm{PhD}$, for selecting primary lymphocytic leukemia cells for the study, and also all medical doctors and nurses involved in collecting patients' samples (Professor Doubek, Dr Brychtova) and all the patients.

Contributors HP and VK prepared the CRISPR/Cas9 knockout clones; HP performed their functional validation. VM prepared CAR T cells, and VM, HP, VK, ALe and ALa conducted all other experiments. JV supervised in vivo studies. TL supervised flow-cytometry measurements. VM, HP, VK and MS discussed and analyzed the data. All authors contributed to the drafting of the manuscript.

Funding This project has received funding from the European Union's (EU) Horizon 2020 research and innovation program under the Marie Skłodowska-Curie actions and is cofinanced by the South Moravian Region under grant agreement number 665860 . This report reflects only the author's view and the EU is not responsible for any use that may be made of the information it contains.

Competing interests None declared.

Patient consent for publication Not required.

Ethics approval The study has been approved by ethics committee of Masaryk University (number of ethics committee case EKV-2017-008). Chronic lymphocytic leukemia samples were selected from the biobank of the Department of Internal Medicine, Hematology and Oncology, where all blood samples are taken after written informed patient's consent, in accordance with the Declaration of Helsinki, under protocols approved by the hospital ethics committee. All animal experiments were performed with the approval of the institute's ethics committee according to the Law on the Protection of Animals Against Cruelty. The research involving animal models has additionally obtained approval by the Ministry of Education, Youth and Sports (MSMT-25883/2017-2).

Provenance and peer review Not commissioned; externally peer reviewed.

Data availability statement Data sharing not applicable as no datasets generated and/or analysed for this study. All data relevant to the study are included in the article or uploaded as supplementary information. No datasets have been generated within this study, and all data relevant to the study have been included in the article figures or supplementary figures.

Open access This is an open access article distributed in accordance with the Creative Commons Attribution Non Commercial (CC BY-NC 4.0) license, which permits others to distribute, remix, adapt, build upon this work non-commercially, and license their derivative works on different terms, provided the original work is properly cited, appropriate credit is given, any changes made indicated, and the use is non-commercial. See http://creativecommons.org/licenses/by-nc/4.0/.

\section{ORCID iD}

Veronika Mancikova http://orcid.org/0000-0002-8155-9927

\section{REFERENCES}

1 Baliakas P, Hadzidimitriou A, Sutton L-A, et al. Recurrent mutations refine prognosis in chronic lymphocytic leukemia. Leukemia 2015;29:329-36.

2 Stilgenbauer S, Schnaiter A, Paschka P, et al. Gene mutations and treatment outcome in chronic lymphocytic leukemia: results from the CLL8 trial. Blood 2014;123:3247-54.

3 Mato AR, Hill BT, Lamanna N, et al. Optimal sequencing of ibrutinib, idelalisib, and venetoclax in chronic lymphocytic leukemia: results from a multicenter study of 683 patients. Ann Oncol 2017;28:1050-6.

4 Kipps TJ, Stevenson FK, CJ W, et al. Correction: chronic lymphocytic leukaemia. Nat Rev Dis Prim 2017;3:16096.

5 Milone MC, Fish JD, Carpenito C, et al. Chimeric receptors containing CD137 signal transduction domains mediate enhanced survival of $\mathrm{T}$ cells and increased antileukemic efficacy in vivo. Mol Ther 2009;17:1453-64.

6 Turtle CJ, Hay KA, Hanafi L-A, et al. Durable molecular remissions in chronic lymphocytic leukemia treated with CD19-specific chimeric antigen receptor-modified T cells after failure of ibrutinib. J Clin Oncol 2017;35:3010-20.

7 Porter DL, Hwang W-T, Frey NV, et al. Chimeric antigen receptor T cells persist and induce sustained remissions in relapsed refractory chronic lymphocytic leukemia. Sci Transl Med 2015;7:303ra139.
8 Maude SL, Laetsch TW, Buechner J, et al. Tisagenlecleucel in children and young adults with B-cell lymphoblastic leukemia. $N$ Engl J Med 2018;378:439-48.

9 Locke FL, Ghobadi A, Jacobson CA, et al. Long-Term safety and activity of axicabtagene ciloleucel in refractory large B-cell lymphoma (ZUMA-1): a single-arm, multicentre, phase 1-2 trial. Lancet Oncol 2019;20:31-42.

10 Lorentzen CL, Straten PT. CD19-Chimeric antigen receptor T cells for treatment of chronic lymphocytic leukaemia and acute lymphoblastic leukaemia. Scand J Immunol 2015;82:307-19.

11 Fraietta JA, Lacey SF, Orlando EJ, et al. Determinants of response and resistance to CD19 chimeric antigen receptor (CAR) T cell therapy of chronic lymphocytic leukemia. Nat Med 2018;24:563-71.

12 Hallek M, Cheson BD, Catovsky D, et al. Guidelines for the diagnosis and treatment of chronic lymphocytic leukemia: a report from the International workshop on chronic lymphocytic leukemia updating the National cancer Institute-Working group 1996 guidelines. Blood 2008;111:5446-56.

13 Brazdilova K, Plevova K, Skuhrova Francova H, et al. Multiple productive IgH rearrangements denote oligoclonality even in immunophenotypically monoclonal CLL. Leukemia 2018;32:234-6.

14 Plevova K, Francova HS, Burckova K, et al. Multiple productive immunoglobulin heavy chain gene rearrangements in chronic lymphocytic leukemia are mostly derived from independent clones. Haematologica 2014;99:329-38.

15 Malcikova J, Tausch E, Rossi D, et al. Eric recommendations for TP53 mutation analysis in chronic lymphocytic leukemia-update on methodological approaches and results interpretation. Leukemia 2018;32:1070-80.

16 Malcikova J, Stano-Kozubik K, Tichy B, et al. Detailed analysis of therapy-driven clonal evolution of TP53 mutations in chronic lymphocytic leukemia. Leukemia 2015;29:877-85.

17 Navrkalova V, Young E, Baliakas P, et al. ATM mutations in major stereotyped subsets of chronic lymphocytic leukemia: enrichment in subset \#2 is associated with markedly short telomeres. Haematologica 2016;101:e369-73.

18 Vavrova E, Kantorova B, Vonkova B, et al. Fragment analysis represents a suitable approach for the detection of hotspot c.7541_7542delCT Notch1 mutation in chronic lymphocytic leukemia. Leuk Res 2017;60:145-50.

19 Strefford JC, Sutton L-A, Baliakas P, et al. Distinct patterns of novel gene mutations in poor-prognostic stereotyped subsets of chronic lymphocytic leukemia: the case of SF3B1 and subset \#2. Leukemia 2013;27:2196-9.

20 Rosén A, Bergh A-C, Gogok P, et al. Lymphoblastoid cell line with B1 cell characteristics established from a chronic lymphocytic leukemia clone by in vitro EBV infection. Oncoimmunology 2012;1:18-27.

21 Stacchini A, Aragno M, Vallario A, et al. MEC1 and MEC2: two new cell lines derived from B-chronic lymphocytic leukaemia in prolymphocytoid transformation. Leuk Res 1999;23:127-36.

22 Imai C, Mihara K, Andreansky M, et al. Chimeric receptors with 4-1BB signaling capacity provoke potent cytotoxicity against acute lymphoblastic leukemia. Leukemia 2004;18:676-84.

23 Oppermann S, Ylanko J, Shi Y, et al. High-Content screening identifies kinase inhibitors that overcome venetoclax resistance in activated CLL cells. Blood 2016;128:934-47.

24 Xue W, Zender L, Miething C, et al. Senescence and tumour clearance is triggered by p53 restoration in murine liver carcinomas. Nature 2007;445:656-60.

25 Ventura A, Kirsch DG, McLaughlin ME, et al. Restoration of p53 function leads to tumour regression in vivo. Nature 2007;445:661-5.

26 Rossi D, Cerri M, Deambrogi C, et al. The prognostic value of TP53 mutations in chronic lymphocytic leukemia is independent of Del17p13: implications for overall survival and chemorefractoriness. Clin Cancer Res 2009;15:995-1004.

27 Landau DA, Tausch E, Taylor-Weiner AN, et al. Mutations driving CLL and their evolution in progression and relapse. Nature 2015;526:525-30.

28 Bertilaccio MTS, Scielzo C, Simonetti G, et al. Xenograft models of chronic lymphocytic leukemia: problems, pitfalls and future directions. Leukemia 2013;27:534-40.

29 Landau DA, Carter SL, Stojanov P, et al. Evolution and impact of subclonal mutations in chronic lymphocytic leukemia. Cell 2013;152:714-26.

30 Guarini A, Marinelli M, Tavolaro S, et al. Atm gene alterations in chronic lymphocytic leukemia patients induce a distinct gene expression profile and predict disease progression. Haematologica 2012;97:47-55.

31 Close V, Close W, Kugler SJ, et al. FBXW7 mutations reduce binding of NOTCH1, leading to cleaved NOTCH1 accumulation and target gene activation in CLL. Blood 2019;133:830-9. 
32 Quentmeier H, Pommerenke C, Ammerpohl O, et al. Subclones in B-lymphoma cell lines: isogenic models for the study of gene regulation. Oncotarget 2016;7:63456-65.

33 Brentjens RJ, Rivière I, Park JH, et al. Safety and persistence of adoptively transferred autologous CD19-targeted T cells in patients with relapsed or chemotherapy refractory B-cell leukemias. Blood 2011;118:4817-28.

34 Porter DL, Levine BL, Kalos M, et al. Chimeric antigen receptormodified T cells in chronic lymphoid leukemia. N Engl J Med 2011;365:725-33.

35 Oscier DG, Gardiner AC, Mould SJ, et al. Multivariate analysis of prognostic factors in CLL: clinical stage, IGVH gene mutational status, and loss or mutation of the p53 gene are independent prognostic factors. Blood 2002;100:1177-84.
36 Austen B, Skowronska A, Baker C, et al. Mutation status of the residual ATM allele is an important determinant of the cellular response to chemotherapy and survival in patients with chronic lymphocytic leukemia containing an 11q deletion. J Clin Oncol 2007;25:5448-57.

37 Yang $\mathrm{CH}$, Wang $\mathrm{Y}$, Sims M, et al. MicroRNA203a suppresses glioma tumorigenesis through an ATM-dependent interferon response pathway. Oncotarget 2017;8:112980-91.

38 Liu X, Li F, Huang Q, et al. Self-Inflicted DNA double-strand breaks sustain tumorigenicity and stemness of cancer cells. Cell Res 2017;27:764-83.

39 Hombach AA, Görgens A, Chmielewski M, et al. Superior Therapeutic Index in Lymphoma Therapy: CD30(+) CD34(+) Hematopoietic Stem Cells Resist a Chimeric Antigen Receptor T-cell Attack. Mol Ther 2016;24:1423-34. 